\title{
Neuropsychological test-based risk prediction of conversion to dementia in amnestic mild cognitive impairment patients: a personal view
}

\author{
Jun Pyo Kim, Hye Min Jang, Hee Jin Kim, Duk L. Na, Sang Won Seo \\ Department of Neurology, Samsung Medical Center, Sungkyunkwan University School of Medicine, Seoul, Korea
}

Received: May 17, 2018

Revised: June 14, 2018

Accepted: June 15, 2018

Corresponding author:

Sang Won Seo

Department of Neurology,

Samsung Medical Center,

Sungkyunkwan University

School of Medicine, 81 Irwon-

ro, Gangnam-gu, Seoul 06351,

Korea

Tel: +82-2-3410-1397

E-mail: sangwonseo@empal. com

This is an Open Access article distributed under the terms of the Creative Commons Attribution Non-Commercial License (http:// creativecommons.org/licenses/ by-nc/4.0/).

\section{ABSTRACT}

The amnestic form of mild cognitive impairment $(\mathrm{aMCl})$ is understood to be a prodromal state of Alzheimer's disease dementia. As recent studies and clinical trials have started to focus on the early detection of and intervention for Alzheimer's disease, aMCI has become an important area of study. Due to the heterogeneous clinical course of $\mathrm{aMCl}$, it is often more challenging for a clinician to predict the prognosis of aMCI patients than of those with Alzheimer's disease dementia patients. Therefore, the ability to predict the clinical course of an aMCl patient based on the patient's clinical data is crucial in both clinical and research settings. In the current study, we present our findings on the structural and prognostic differences between $\mathrm{aMCl}$ and Alzheimer's disease dementia according to neuropsychological test results. Additionally, we introduce a neuropsychological test-based risk prediction model of the conversion to dementia.

Keywords: Alzheimer disease; Amnestic mild cognitive impairment; Prediction model

\section{INTRODUCTION}

The amnestic form of mild cognitive impairment $(\mathrm{aMCl})$ is regarded as a prodromal state of Alzheimer's disease (AD) dementia [1]. According to the results of neuropsychological tests, aMCI can be classified in several ways: single-domain vs. multi-domain, verbal memory-versus visual memory-predominant, and early vs. late [2-4]. In addition to the patterns found in the neuropsychological test results, aMCI patients show varying degrees of brain atrophy and clinical course. While some patients remain stable or even revert to normal cognition, the annual rate of the conversion to dementia for aMCl patients is up to $20 \%$, which is suggestive of a heterogeneous entity $[2,5,6]$. Therefore, understanding the clinical course of aMCl and determining the prognostic factors of the conversion to dementia have attracted considerable attention.

Regarding the conversion from aMCI to $\mathrm{AD}$ dementia, several studies have reported demographical, radiological, neuropsychological, and laboratory factors predictive of the progression. Poor performance in episodic memory tests, cognitive impairment in other domains such as frontal/executive function, and depression are all known to predict the conversion to dementia in aMCl subjects [7-10]. In fact, even among aMCl subjects, those with more severe 
memory impairment, multi-domain cognitive deficits, or verbal memory impairment have higher dementia conversion rates than those with milder memory impairment, memory involvement only, or visual memory impairment only [3,11-13]. In addition, the results of a recent meta-analysis showed that older age, female gender, hypertension, diabetes, hippocampal and medial temporal lobe atrophy, entorhinal atrophy, lower mini-mental status examination (MMSE) score, higher Alzheimer's Disease Assessment Scale-cognitive subscale (ADAS-cog) score, presence of the apolipoprotein E4 (ApoE4) allele, and abnormal cerebrospinal fluid tau levels are associated with the progression from $\mathrm{aMCl}$ to $\mathrm{AD}$ dementia [14]. Additionally, aMCl patients with positive amyloid positron emission tomography (PET) scans are significantly more likely to convert to dementia than amyloid-negative patients [15].

While the above-mentioned studies have shown differences at the group level, they did not predict the risk of dementia at the individual level. Although group analysis provides perspective in understanding the nature of the disease entity, it is not enough to predict an individual's risk. Therefore, it is crucial to develop risk prediction models such as nomograms, which are useful for patient counseling, management, risk stratification, and sometimes for the determination of early enrollment in clinical trials [16]. In a recent study by our group, we developed a risk prediction model to predict the risk of dementia in aMCl patients using clinical features and neuropsychological results. Previous studies have incorporated imaging, genetic, and cerebrospinal fluid biomarkers into prediction models. However, biomarker studies are relatively expensive and sometimes unavailable depending on the clinical situation. Our risk prediction model based on neuropsychological tests is useful because neuropsychological test results can be easily obtained in any clinical setting. This paper highlights our recent study findings on the risk differences in the conversion to dementia according to neuropsychological test results and as well as the risk prediction model developed by our group.

\section{STRUCTURAL AND PROGNOSTIC DIF- FERENCES ACCORDING TO NEUROPSY- CHOLOGICAL TEST RESULTS}

\section{Single-domain versus multi-domain}

It is common to classify aMCl into single-domain versus multi-domain [2]. The results from our magnetic resonance imaging (MRI) study suggested that there is a hierarchical re- lationship between single-domain aMCI (single-aMCI) and multi-domain aMCI (multi-aMCI) [17]. Compared with normal controls (NCs), single-aMCl patients showed cortical thinning in the left medial temporal lobe and multi-aMCl patients showed cortical thinning in the left temporal lobe, precuneus, and the anterior and inferior basal temporal and insular and temporal association cortices. Additionally, multi-aMCI patients had significant cortical thinning in the left precuneus when compared with single-aMCl patients.

In line with the structural difference between single-aMCI and multi-aMCl patients, there was significant difference in the risk of conversion to dementia between the two groups [18]. Multi-aMCl patients had a significantly higher dementia risk than single-aMCI patients (odds ratio [OR], 3.60; 95\% confidence interval [Cl], 1.78 to 7.29 ). These results show that there are structural and prognostic differences and suggest that multi-aMCI lies between single-aMCI and $A D$ dementia.

\section{Visual vs. verbal memory impairment}

Both verbal and visual memory tests are used in the diagnosis of aMCl $[5,19]$. To determine if there is a structural difference in the brain depending on the type of impairment (i.e., visual or verbal memory), we compared the topographic pattern of cortical thinning among three subtypes of aMCl: $\mathrm{aMCl}$ with predominant verbal memory impairment (verbal-aMCI), $\mathrm{aMCl}$ with predominant visual memory impairment (visu$\mathrm{al}-\mathrm{aMCl}$ ), and aMCl with both verbal and visual memory predominant impairment (both-aMCl) [20]. Patients with verbal-aMCl showed significant cortical thinning in the left anterior and medial temporal regions compared with NC, while visual-aMCl patients did not. Furthermore, the cortical thinning pattern of the both-aMCl group included that found in the verbal-aMCl group, but was more widespread, with involvement in the bilateral temporal regions.

In terms of clinical course, we evaluated the longitudinal outcomes of aMCl patients according to the modality of the memory impairment involved [13]. Patients were classified into visual-aMCI, verbal-aMCl, and both-aMCI groups, as described above. When compared with the visual-aMCI group, the verbal-aMCl (OR, 2.09; 95\% Cl, 1.26 to $3.47 ; \mathrm{P}=0.004)$ and both-aMCl (OR, 2.94; 95\% Cl, 1.89 to 4.55; $\mathrm{P}<0.001)$ groups exhibited a higher risk of progression to dementia. Interestingly, recognition failure was associated with an increased risk of progression to dementia only in the visual-aMCl group. These results suggest that visual-aMCI patients might represent a combination of heterogeneous subgroups and that recognition failure is a crucial component in the identifi- 
cation of patients with true AD pathology. In contrast, verbal-aMCl and both-aMCl are precursors to $A D$ regardless of the presence or absence of recognition failure.

\section{Early-stage vs. late-stage aMCl}

aMCI patients can be classified into early-stage (E-aMCI) and late-stage (L-aMCI) according to memory test scores [3]. The concept of $\mathrm{E}-\mathrm{aMCl}$ is crucial in terms of the early enrollment of aMCl patients into clinical trials. To evaluate whether $\mathrm{E}-\mathrm{aMCl}$ may represent a very early stage of $\mathrm{AD}$, we compared the patterns of cortical thinning between $\mathrm{E}-\mathrm{aMCl}$ and $\mathrm{L}-\mathrm{aMCl}$ patients [21]. aMCI patients were divided into an $\mathrm{E}-\mathrm{aMCl}$ group (scores between -1.5 standard deviations [SD] and -1.0 $\mathrm{SD}$ from the mean) and an $\mathrm{L}-\mathrm{aMCl}$ group (scores lower than -1.5 SD from the mean). Compared with NC, the E-aMCI group showed significant cortical thinning in the left medial temporal and insular regions. By contrast, the L-aMCl group showed cortical thinning in more widespread regions, including the bilateral dorsolateral prefrontal, and anterior and medial temporal and temporoparietal association cortices, as well as the precuneus. The presence and location of atrophy in the $\mathrm{E}-\mathrm{aMCl}$ group suggests that $\mathrm{E}-\mathrm{aMCl}$ represents a true pathological state of very early $A D$, since the medial temporal and insular regions are known to be involved in the earliest stage of $A D$ pathology [22]. The widespread pattern of cortical thinning in $\mathrm{L}-\mathrm{aMCl}$ patients and the similarity of the atrophy patterns with that of $A D$ dementia $[23,24]$ suggest that L-aMCl may be a transitional state between $\mathrm{E}-\mathrm{aMCl}$ and $A D$ dementia.

In a multi-center study by our group, the clinical course of $\mathrm{E}-\mathrm{aMCl}$ and $\mathrm{L}-\mathrm{aMCl}$ groups was compared [25]. The annual conversion rate was $10.6 \%$ in the $\mathrm{E}-\mathrm{aMCl}$ and $20.2 \%$ in the $\mathrm{L}$-aMCI group. Cox analysis revealed that the risk of $A D$ conversion was higher in $\mathrm{L}-\mathrm{aMCl}$ than in $\mathrm{E}-\mathrm{aMCl}$ (hazard ratio, 2.26; $P=0.02$ ) after controlling for age, gender, and baseline MMSE score.

\section{DEVELOPMENT OF A PREDICTION MOD- EL OF THE CONVERSION TO DEMENTIA}

A nomogram is designed to help clinicians and patients in risk assessment and predicting outcomes of treatment based on specific patient and disease characteristics [16]. They can easily be applied in complex and busy clinical environments due to their advantages of the involvement of visual displays and their simplicity.

Based on the results of group analyses, we developed a no-
Points

$\begin{array}{lllllllllll}0 & 10 & 20 & 30 & 40 & 50 & 60 & 70 & 80 & 90 & 100\end{array}$

Age

Modality

$\begin{array}{llllllllll}45 & 50 & 55 & 60 & 65 & 70 & 75 & 80 & 85 & 90\end{array}$

Visual $\stackrel{\text { Berbal Both }}{\longrightarrow}$

Severity

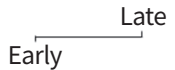

Multiplicity

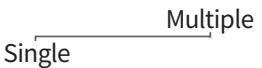

Total points

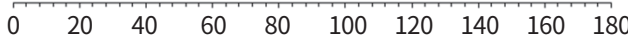

Predicted probability

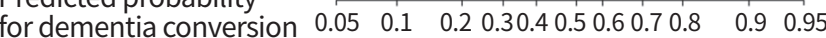

Fig. 1. Nomogram for predicting the conversion to dementia.

mogram to predict the risk of the conversion to dementia in $\mathrm{aMCl}$ patients using clinical features and neuropsychological results [18]. A total of $338 \mathrm{aMCl}$ patients were included in the analysis. The results of a multivariable logistic regression showed that individuals with both-aMCI $(\mathrm{OR}, 4.30 ; 95 \% \mathrm{CI}$, 1.95 to 9.47$)$, $\mathrm{L}$-aMCI (OR, $2.15 ; 95 \% \mathrm{Cl}, 1.06$ to 4.36$)$, and multiple-aMCI (OR, 3.60; $95 \% \mathrm{Cl}, 1.78$ to 7.29$)$ all had a significantly higher dementia risk than those with visual-aMCI, $\mathrm{E}-\mathrm{aMCl}$, and single-aMCl, respectively. The prediction performance (C-statistics) of our model was 0.77 in the original data and $0.75 / 0.79$ in the internal/external validation. The nomogram is shown in Fig. 1. A specific point was matched to each variable based on the beta coefficients from the regression analyses. The total points, determined from the sum of each point, were used to indicate the overall risk score.

\section{DISCUSSION}

Recently, aMCl has become regarded not just as a transitional state between normal cognition and AD dementia, but also as the most crucial stage for studies and clinical trials. In clinical practice, aMCl patients require more detailed counseling than demented patients due to the uncertainties in their clinical outcomes. Therefore, understanding the natural course of aMCl and predicting its prognosis is important in both research and clinical aspects.

Our studies on the structural changes occurring in aMCI patients have provided us with some perspectives on the hierarchical orders of aMCl. Patients with multi-aMCl, both-aMCI, 
and $\mathrm{L}-\mathrm{aMCl}$ showed more widespread cortical thinning patterns than patients with single-aMCl, verbal-aMCl, or E-aMCI $[17,20,21]$. However, structural changes were also detected in single-a $\mathrm{MCl}$, verbal-aMCl, and $\mathrm{E}-\mathrm{aMCl}$ patients, and the patterns of cortical thinning were consistent with the known early involvement of $A D$ pathology. These results suggest that on the spectrum from NC to AD dementia, multi-aMCl, both-aMCl, and L-aMCl lie closer to AD dementia than single-aMCl, visual-aMCl, and E-aMCl. The hierarchical order between both-aMCl and verbal-aMCl seems clear, but because the visual-aMCl patients did not show significant cortical atrophy, more evidence is needed before conclusions can be drawn regarding visual-aMCl.

The conversion from $\mathrm{aMCl}$ to dementia might be the most important issue for aMCl patients. Results from our group have shown that multi-aMCl, verbal-aMCl, both-aMCl, L-aM$\mathrm{Cl}$, and positive amyloid PET scans are predictors of dementia conversion in aMCl patients $[13,25]$. Until recently, most studies on aMCl have been group-based analyses. To be able to readily apply our study results in clinical practice, we have developed a prediction model in the form of a nomogram to predict the 3-year risk of conversion to dementia in aMCl patients. The risk prediction model showed fair discrimination (C-statistics, 0.77) and calibration capabilities.

In previous studies, several prediction models using biomarker information such as the levels of $A \beta$ in the cerebrospinal fluid, amyloid deposition on PET, and atrophy measured on brain MRI have been created [26-29]. However, biomarker studies are relatively expensive, difficult to conduct, and are sometimes unavailable depending on the clinical situation. In addition, data from advanced biomarker studies sometimes require complicated processing procedures that clinical sites may not be able to perform. Our prediction model is useful in clinical settings because it is based on neuropsychological test results that are easy to obtain and that do not require complex preprocessing.

\section{CONCLUSION}

Research on aMCl has been transformed due to the recent advances in imaging technology and the accumulation of patient data. Amyloid PET scans are increasingly used in clinical settings, and tracers for tau PET are being developed. Additionally, machine-learning techniques have enabled for automated image analysis at the individual level. However, these state-of-the-art technologies have both advantages and disadvantages. These technologies make more accurate diagnoses and the individualization of treatment possible but are prone to non-medical problems such as high costs and poor availability. Fortunately, our results regarding prediction models demonstrate that decent predictive accuracy can be achieved from readily accessible data. The key to future research in the field of dementia might be the incorporation of advanced techniques into research models as well as ensuring such models are ready and available for use in clinical practice.

\section{CONFLICTS OF INTEREST}

No potential conflict of interest relevant to this article was reported.

\section{ACKNOWLEDGMENTS}

This work was supported by the National Research Foundation of Korea (NRF) grant funded by the Korea government (MSIP) (No. NRF-2017R1A2B2005081).

\section{REFERENCES}

1. Petersen RC, Doody R, Kurz A, Mohs RC, Morris JC, Rabins $\mathrm{PV}$, et al. Current concepts in mild cognitive impairment. Arch Neurol 2001;58:1985-92.

2. Busse A, Hensel A, Guhne U, Angermeyer MC, Riedel-Heller SG. Mild cognitive impairment: long-term course of four clinical subtypes. Neurology 2006;67:2176-85.

3. Aisen PS, Petersen RC, Donohue MC, Gamst A, Raman R, Thomas RG, et al. Clinical core of the Alzheimer's disease neuroimaging initiative: progress and plans. Alzheimers Dement 2010;6:239-46.

4. Guo Q, Zhao Q, Chen M, Ding D, Hong Z. A comparison study of mild cognitive impairment with 3 memory tests among Chinese individuals. Alzheimer Dis Assoc Disord 2009;23:253-9.

5. Larrieu S, Letenneur L, Orgogozo JM, Fabrigoule C, Amieva $\mathrm{H}$, Le Carret N, et al. Incidence and outcome of mild cognitive impairment in a population-based prospective cohort. Neurology 2002;59:1594-9.

6. Petersen RC. Mild cognitive impairment as a diagnostic entity. J Intern Med 2004;256:183-94.

7. Tabert MH, Manly JJ, Liu X, Pelton GH, Rosenblum S, Jacobs M, et al. Neuropsychological prediction of conversion to Alzheimer disease in patients with mild cognitive impairment. Arch Gen Psychiatry 2006;63:916-24. 
8. Blacker D, Lee H, Muzikansky A, Martin EC, Tanzi R, McArdle JJ, et al. Neuropsychological measures in normal individuals that predict subsequent cognitive decline. Arch Neurol 2007;64:862-71.

9. Amieva H, Letenneur L, Dartigues JF, Rouch-Leroyer I, Sourgen C, D'Alchee-Biree F, et al. Annual rate and predictors of conversion to dementia in subjects presenting mild cognitive impairment criteria defined according to a population-based study. Dement Geriatr Cogn Disord 2004;18:87-93.

10. Palmer K, Berger AK, Monastero R, Winblad B, Backman L, Fratiglioni L. Predictors of progression from mild cognitive impairment to Alzheimer disease. Neurology 2007; 68:1596-602.

11. Fazekas F. Magnetic resonance signal abnormalities in asymptomatic individuals: their incidence and functional correlates. Eur Neurol 1989;29:164-8.

12. Morris JC, Storandt M, Miller JP, McKeel DW, Price JL, Rubin $\mathrm{EH}$, et al. Mild cognitive impairment represents early-stage Alzheimer disease. Arch Neurol 2001;58:397-405.

13. Ye BS, Chin J, Kim SY, Lee JS, Kim EJ, Lee Y, et al. The heterogeneity and natural history of mild cognitive impairment of visual memory predominant type. J Alzheimers Dis 2015;43:143-52.

14. Li JQ, Tan L, Wang HF, Tan MS, Tan L, Xu W, et al. Risk factors for predicting progression from mild cognitive impairment to Alzheimer's disease: a systematic review and meta-analysis of cohort studies. J Neurol Neurosurg Psychiatry 2016;87:476-84.

15. Okello A, Koivunen J, Edison P, Archer HA, Turkheimer FE, Nagren $\mathrm{K}$, et al. Conversion of amyloid positive and negative $\mathrm{MCl}$ to AD over 3 years: an 11C-PIB PET study. Neurology 2009;73:754-60.

16. Fu AZ, Cantor SB, Kattan MW. Use of nomograms for personalized decision-analytic recommendations. Med Decis Making 2010;30:267-74.

17. Seo SW, Im K, Lee JM, Kim YH, Kim ST, Kim SY, et al. Cortical thickness in single- versus multiple-domain amnestic mild cognitive impairment. Neuroimage 2007;36:289-97.

18. Jang H, Ye BS, Woo S, Kim SW, Chin J, Choi SH, et al. Prediction model of conversion to dementia risk in subjects with amnestic mild cognitive impairment: a longitudinal, multi-center clinic-based study. J Alzheimers Dis 2017;60: 1579-87.

19. Geslani DM, Tierney MC, Herrmann N, Szalai JP. Mild cog- nitive impairment: an operational definition and its conversion rate to Alzheimer's disease. Dement Geriatr Cogn Disord 2005;19:383-9.

20. Kim MJ, Im K, Lee JM, Park A, Chin J, Kim GH, et al. Cortical thinning in verbal, visual, and both memory-predominant mild cognitive impairment. Alzheimer Dis Assoc Disord 2011;25:242-9.

21. Ye BS, Seo SW, Yang JJ, Kim HJ, Kim YJ, Yoon CW, et al. Comparison of cortical thickness in patients with early-stage versus late-stage amnestic mild cognitive impairment. Eur J Neurol 2014;21:86-92.

22. Braak H, Braak E. Neuropathological stageing of Alzheimer-related changes. Acta Neuropathol 1991;82:239-59.

23. Dickerson BC, Bakkour A, Salat DH, Feczko E, Pacheco J, Greve DN, et al. The cortical signature of Alzheimer's disease: regionally specific cortical thinning relates to symptom severity in very mild to mild $A D$ dementia and is detectable in asymptomatic amyloid-positive individuals. Cereb Cortex 2009;19:497-510.

24. Du AT, Schuff N, Kramer JH, Rosen HJ, Gorno-Tempini ML, Rankin K, et al. Different regional patterns of cortical thinning in Alzheimer's disease and frontotemporal dementia. Brain 2007;130(Pt 4):1159-66.

25. Ye BS, Seo SW, Cho H, Kim SY, Lee JS, Kim EJ, et al. Effects of education on the progression of early- versus latestage mild cognitive impairment. Int Psychogeriatr 2013; 25:597-606.

26. Ewers M, Buerger K, Teipel SJ, Scheltens P, Schroder J, Zinkowski RP, et al. Multicenter assessment of CSF-phosphorylated tau for the prediction of conversion of $\mathrm{MCl}$. Neurology 2007;69:2205-12.

27. Ewers M, Walsh C, Trojanowski JQ, Shaw LM, Petersen $\mathrm{RC}$, Jack CR Jr, et al. Prediction of conversion from mild cognitive impairment to Alzheimer's disease dementia based upon biomarkers and neuropsychological test performance. Neurobiol Aging 2012;33:1203-14.

28. Lopez ME, Turrero A, Cuesta P, Lopez-Sanz D, Bruna R, Marcos A, et al. Searching for primary predictors of conversion from mild cognitive impairment to Alzheimer's disease: a multivariate follow-up study. J Alzheimers Dis 2016;52:133-43.

29. Westman E, Muehlboeck JS, Simmons A. Combining MRI and CSF measures for classification of Alzheimer's disease and prediction of mild cognitive impairment conversion. Neuroimage 2012;62:229-38. 